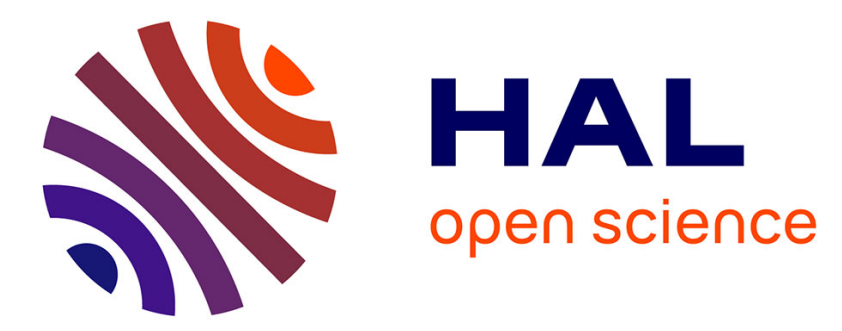

\title{
Étude de cycles intermittents à adsorption solide pour la réfrigération solaire
}

\author{
J.J. Guilleminot, Frédéric Meunier, B. Mischler
}

\section{To cite this version:}

J.J. Guilleminot, Frédéric Meunier, B. Mischler. Étude de cycles intermittents à adsorption solide pour la réfrigération solaire. Revue de Physique Appliquée, 1980, 15 (3), pp.441-452. 10.1051/rphysap:01980001503044100 . jpa-00244751

\section{HAL Id: jpa-00244751 https://hal.science/jpa-00244751}

Submitted on 1 Jan 1980

HAL is a multi-disciplinary open access archive for the deposit and dissemination of scientific research documents, whether they are published or not. The documents may come from teaching and research institutions in France or abroad, or from public or private research centers.
L'archive ouverte pluridisciplinaire HAL, est destinée au dépôt et à la diffusion de documents scientifiques de niveau recherche, publiés ou non, émanant des établissements d'enseignement et de recherche français ou étrangers, des laboratoires publics ou privés. 


\title{
Étude de cycles intermittents à adsorption solide pour la réfrigération solaire
}

\author{
J J Guilleminot, F Meunier et B. Mischler (*) \\ Laboratoire de Physique des Solides (**), Bât. 510, Université Paris-Sud, Centre d'Orsay, 91405 Orsay, France
}

(Reçu le 27 juillet 1979, révisé le 21 novembre 1979, accepté le 26 novembre 1979)

\begin{abstract}
Résumé. - Nous présentons les résultats d'une analyse thermodynamique des machines quadrithermes à simple effet ainsi que les résultats des calculs des performances de machines utilisant des cycles intermittents à adsorption solide pour la réfrigération solaire. L'influence des différents paramètres est discutée et une comparaison avec le cycle LiBr-eau est effectuée.
\end{abstract}

\begin{abstract}
We present the results of a thermodynamical analysis of sorption machines as well as the results of the calculation of the COP of cycles using desiccants for solar cooling. The effect of various parameters is discussed and a comparison with $\mathrm{LiBr}-\mathrm{H}_{2} \mathrm{O}$ is performed.
\end{abstract}

1. Introduction. - Le problème général de la réfrigération en utilisant un cycle thermodynamique est celui du transfert de chaleur (par lintermédiaire d'un transfert de masse) d'une basse température (évaporateur) à une température plus élevée (absorbeur ou condenseur)

Pour que ce transfert soit possible, on doit avoir recours à une source d'énergie qui peut être, soit :

- de l'énergie mécanique (cycles à compression),

- de l'énergie interne (cycles à sorption).

Cette énergie (mécanique ou interne) peut provenir elle-même de la conversion thermodynamique de l'énergie solaire.

Nous voyons deux avantages aux cycles à sorption sur les cycles à compression [1] :

- ils peuvent fonctionner sans utiliser aucune partie mobile,

- à basse température de la source chaude, leur rendement n'est pas très éloigné du rendement idéal de Carnot.

\section{Considérations thermodynamiques. -2.1}

MACHINE IDÉAlE A TROIS TEMPÉRATURES. - Une machine idéale à trois températures se décompose en deux machines à deux températures couplées via l'énergie mécanique. L'une des deux machines sert à produire l'énergie mécanique que l'autre utilise pour

*) Département de Mathématiques, Université de Paris-Sud, 91405 Orsay.

$\left.{ }^{* *}\right)$ Laboratoire associé au C.N.R.S produire le froid; les flux de chaleur et d'énergie sont indiqués sur la figure 1 et le rendement idéal de chaque machine étant donné par le principe de Carnot, on obtient le résultat bien connu pour le coefficient de performance $(C O P)$ thermique total :

$$
C O P=\frac{Q_{\mathrm{ev}}}{Q_{\mathrm{reg}}}=\frac{T_{\mathrm{ev}}\left(T_{\mathrm{reg}}-T_{0}\right)}{T_{\mathrm{reg}}\left(T_{0}-T_{\mathrm{ev}}\right)}
$$

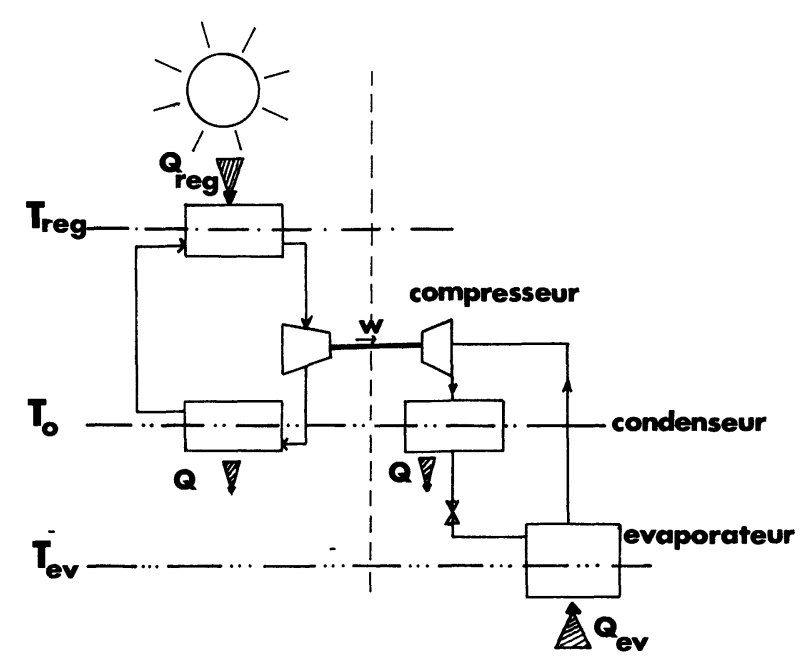

Fig. 1. - Machine à trois températures correspondant au couplage de deux cycles de Carnot idéaux.

[Three temperature levels machine working with two ideal Carnot cycles.] 
où

- $Q_{\text {ev }}$ est la chaleur extraite à l'évaporateur,

- $Q_{\text {reg }}$ est la chaleur fournie à la source chaude,

- $T_{\mathrm{ev}}$ est la température de l'évaporateur,

- $T_{0}$ est la température de la source froide,

- $T_{\text {reg }}$ est la température de la source chaude.

Le coefficient de performance est donc une fonction croissante de la température de la source chaude (Fig. 3) tendant asymptotiquement vers $\frac{T_{\mathrm{ev}_{\mathrm{v}}}}{T_{0}-T_{\mathrm{ev}}}$ qui peut être très supérieur à l'unité

\subsection{MACHINE A SORPTION A QUATRE TEMPÉRATURES.} - Une machine à sorption correspond au couplage de deux machines à deux températures fonctionnant avec le même fluide condensable et sans conversion en énergie mécanique. Une analyse thermodynamique est présentée dans l'annexe. Résumons ici le fonctionnement : dans l'étape de régénération on transfère une molécule de la phase sorbée à la température de la source chaude $\left(T_{\text {reg }}\right)$ vers la phase liquide à température ambiante ; ce transfert correspond à une production d'énergie interne $U_{0}$, bien définie (provenant de la différence d'énergie de liaison en phase adsorbée et en phase liquide). Dans l'étape de production de froid, on veut transférer cette molécule de la phase liquide à basse température $\left(T_{\mathrm{ev}}\right)$ vers la phase sorbée à température ambiante. Trois cas se présentent (Fig. 2) :

- l'énergie interne produite $U_{0}\left({ }^{1}\right)$ lors de la régénération est inférieure à l'énergie nécessaire pour ce transfert et ce transfert n'est pas possible. D'où la notion de température seuil au moment de la régénération;

- l'énergie interne $U_{0}$ correspond exactement à l'énergie nécessaire au transfert, on a en fait une

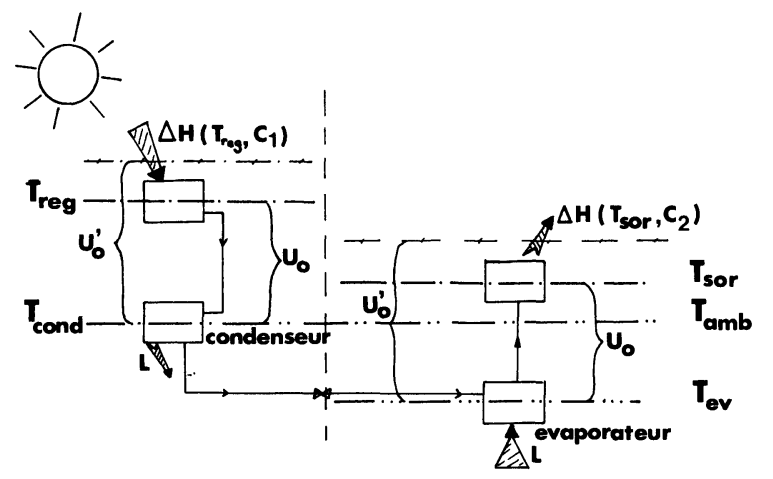

Fig. 2. - Machine à sorption à quatre températures.

[Four temperature levels machine.]

( ${ }^{1}$ L Lénergie interne $U_{0}$ produite peut être visualisée sous la forme d'un site libéré dans un adsorbant solide lors de la régénération et qui sera réoccupé lors de la sorption. machine tritherme dont le rendement idéal sera le COP de Carnot;

- l'énergie interne $U_{0}$ est supérieure à l'énergie nécessaire au transfert et alors on peut transférer la molécule à une température supérieure à la température ambiante

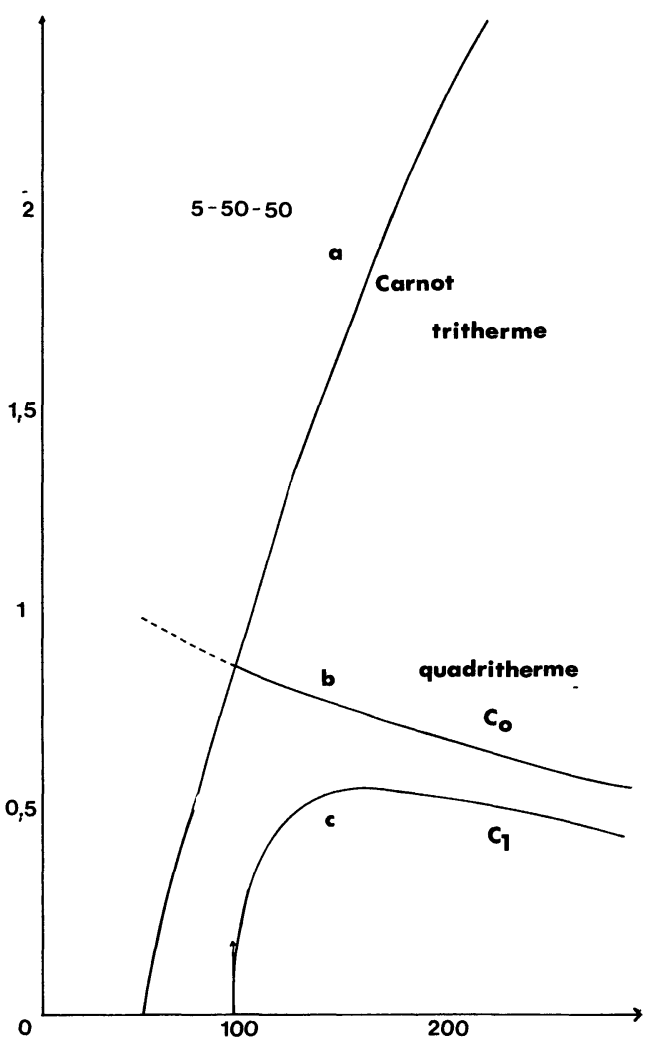

Fig. 3. - Courbes de COP. a) Carnot $T_{\mathrm{ev}}=5, T_{0}=50^{\circ} \mathrm{C}$. h) $C_{0}$ d'une machine quachitherme $T_{\mathrm{ev}}=5, T_{\text {cond }}=T_{\mathrm{ads}}=50$ c) $C_{1}$ de la même machine.

[COP curves. a) Carnot $\left.T_{\mathrm{ev}}=5, T_{0}=50^{\circ} \mathrm{C} h\right) C_{0}$ for a four temperature levels machine with $T_{\mathrm{ev}}=5^{\circ} \mathrm{C}$ and $T_{\text {cond }}=T_{\text {ads }}=50^{\circ} \mathrm{C}$ c) $C_{1}$ for the same machine.]

Qualitativement, nous venons d'introduire deux notions importantes :

2.2.1 Température seuil. - A la grandeur finie $T_{\mathrm{amb}}-T_{\mathrm{ev}}$ va correspondre une autre quantité finie $T_{\text {seuil }}-T_{\text {amb }}$ où $T_{\text {seuil }}$ est la température minimale que doit atteindre le régénérateur pour que le cycle fonctionne. Cette notion de température seuil n'existe pas dans le cas de machines à trois températures (pour une machine à trois températures utilisant un gaz parfait la démultiplication est théoriquement totale entre l'énergie produite dans une phase et l'énergie utilisée dans l'autre phase, ce qui n'est pas le cas des machines à sorption).

2.2.2 Températures couplées. - La température de la source chaude et la température à laquelle peut 
se produire la sorption sont couplées par l'équation suivante (cf. annexe)

$$
\Delta H\left(\frac{1}{T_{\text {reg }}}-\frac{1}{T_{\text {sorp }}}\right)=L\left(\frac{1}{T_{\text {cond }}}-\frac{1}{T_{\text {ev }}}\right)
$$

où

- $\Delta H$ est l'enthalpie de désorption,

- $L$ est la chaleur latente d'évaporation du fluide,

- $T_{\text {reg }}$ est la température de régénération,

- $T_{\text {cond }}$ est la température du condenseur,

- $T_{\text {ev }}$ est la température de l'évaporateur,

- $T_{\text {sor }}$ est la température de sorption.

La variation de $T_{\text {sor }}$ en fonction de $T_{\text {reg }}$ est portée sur la figure 4 pour différentes valeurs de $\Delta H / L$.

Que se passe-t-il si on fait fonctionner la machine quadritherme en machine tritherme (c'est-à-dire en imposant la température ambiante comme température de sorption) ? On crée une situation d'écart à l'état stationnaire et il y a production d'entropie due à l'irréversibilité au moment de la sorption; cette production d'entropie, correspondant à une sorption effectuée à $T_{\mathrm{amb}}$ et non à $T_{\text {sor }}$ donnée par l'équation (2), est égale à :

$$
\Delta_{\mathrm{i}} S=\Delta T\left(\frac{1}{T_{\mathrm{amb}}}-\frac{1}{T_{\mathrm{sor}}}\right)
$$

Cette production d'entropie sera bénéfique pour la cinétique de la sorption mais maléfique pour le $C O P$.

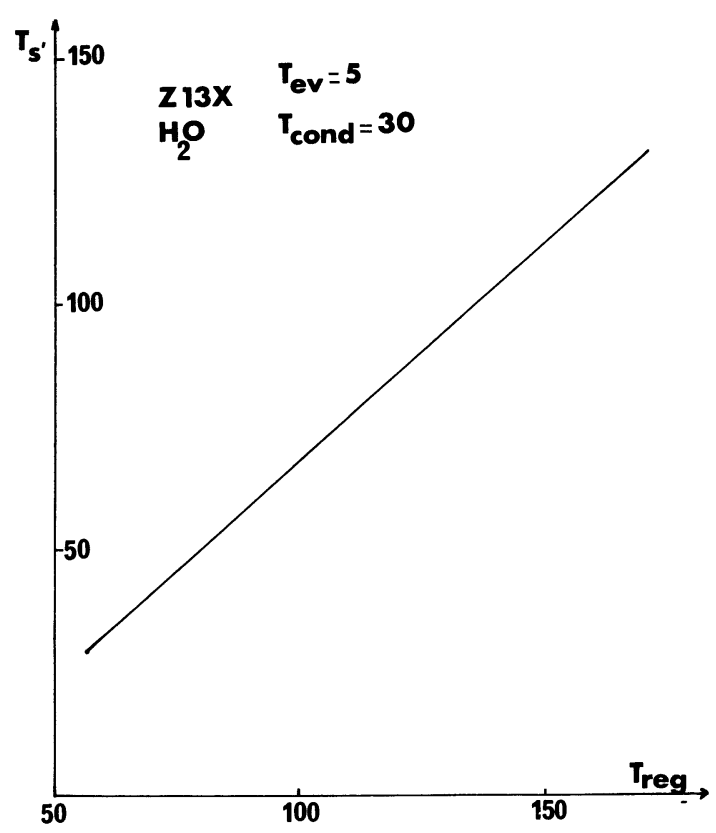

Fig. 4. - Variation de $T_{\checkmark}$, température à laquelle commence ladsorption après la régénération, en fonction de la température de régénération pour $T_{\mathrm{ev}}=5^{\circ} \mathrm{C}$ et $T_{\text {cond }}=30^{\circ} \mathrm{C}$.

[Variation of $T_{\mathrm{s}^{\prime}}$, temperature at which begins the adsorption after the regeneration, as a function of the regeneration temperature for $T_{\text {ev }}=5^{\circ} \mathrm{C}$ and $T_{\text {cond }}=30^{\circ} \mathrm{C}$.]
Le $C O P$ thermique idéal d'une machine à sorption n'est plus une fonction croissante de la température de la source chaude mais au contraire c'est une fonction à marche présentant un maximum à la température seuil $T_{\mathrm{s}}$ (Fig. 3).

Les considérations présentées plus haut concernent les machines simples dans lesquelles toute la chaleur correspondant à la désorption est fournie par la source chaude extérieure. Il est évident qu'en utilisant des machines à étage avec récupération de la chaleur de sorption, il est nécessaire de modifier le raisonnement.

3. Cycle fermé à adsorption solide à effet simple. Dans cet article, nous nous plaçons dans une perspective de technologie à basse température de captation de l'énergie solaire et sans récupération de chaleur dans le cycle. Des études sont en cours sur des cycles à effet multiple avec récupération de chaleur devant conduire à une technologie plus sophistiquée.

Nous allons d'abord décrire le cycle à effet simple et ensuite décrire la machine.

3.1 DESCRIPTION DU CYCLE ZOLITHE $13 \mathrm{X}-\mathrm{H}_{2} \mathrm{O}$ [2] - Un réseau d'isostères du couple zéolithe $13 \mathrm{X}-\mathrm{H}_{2} \mathrm{O}$ (déterminé expérimentalement par thermogravimétrie [3]) est présenté sur la figure 5. La température de la zéolithe est portée en abscisses, alors qu'en ordonnées est portée la température de l'eau dont les vapeurs sont en équilibre avec la phase adsorbée. Les isostères correspondent à des masses adsorbées différentes (les nombres indiqués correspondent à des grammes d'eau adsorbée par kilogramme de zéolithe anhydre).

Deux cycles sont représentés sur la figure 5, l'un

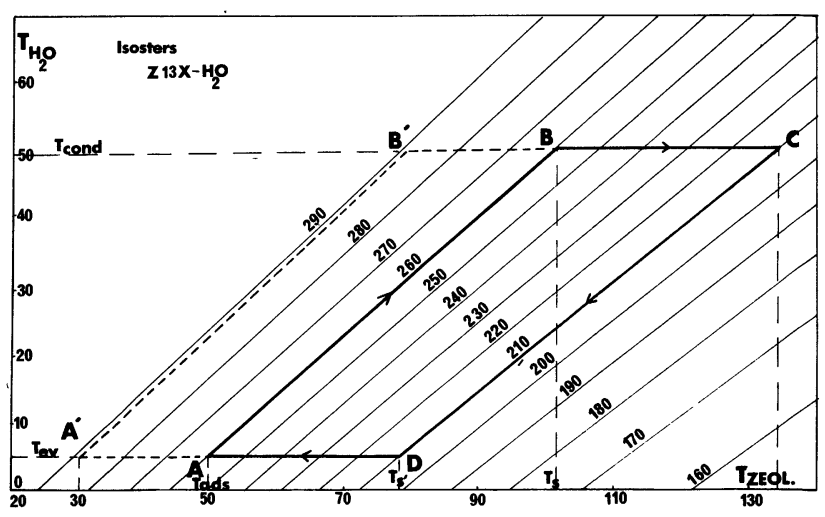

Fig. 5. - Isostères du couple zéolithe $13 \mathrm{X}-\mathrm{H}_{2} \mathrm{O}$. Les nombres sur les isostères indiquent la quantité d'eau adsorbée en grammes d'eau par kilogramme de zéolithe anhydre. ABCD représente un cycle de réfrigération avec $T_{\mathrm{ev}}=5^{\circ} \mathrm{C}, T_{\text {cond }}=T_{\mathrm{ads}}=50^{\circ} \mathrm{C}$ et $T_{\text {reg }}=130^{\circ} \mathrm{C}$. $\mathrm{A}^{\prime} \mathrm{B}^{\prime} \mathrm{CD}$ représente un cycle de réfrigération avec $T_{\text {ads }}=30^{\circ} \mathrm{C}$ au lieu de $50^{\circ} \mathrm{C}$.

[Isosteres for the zeolithe $13 \mathrm{X}-\mathrm{H}_{2} \mathrm{O}$ pair. The numbers on the isosteres give amount of sorbed water in grammes of water per kilogramme of anhydrous zeolithe. ABCD is a cooling cycle with $T_{\mathrm{ev}}=5^{\circ} \mathrm{C}, T_{\text {cond }}=T_{\text {ad }} \quad 50^{\circ} \mathrm{C}$ and $T_{\text {reg }}=130^{\circ} \mathrm{C} . \mathrm{A}^{\prime} \mathrm{B}^{\prime} \mathrm{CD}$ is another cycle with $T_{\text {.1 }}=30^{\circ} \mathrm{C}$ instead of $50^{\circ} \mathrm{C}$.] 
ABCD correspond aux conditions de températures suivantes :

$$
\begin{aligned}
& T_{\text {ev }}=5{ }^{\circ} \mathrm{C} \\
& T_{\text {cond }}=50^{\circ} \mathrm{C} \\
& T_{\text {ads }}=50^{\circ} \mathrm{C} \\
& T_{\text {reg }}=130^{\circ} \mathrm{C}
\end{aligned}
$$

l'autre $\mathrm{A}^{\prime} \mathrm{B}^{\prime} \mathrm{CD}$ correspond à $T_{\text {ads }}=30^{\circ} \mathrm{C}$ au lieu de $50^{\circ} \mathrm{C}$

La température seuil $T_{\mathrm{s}}$ est donnée par l'abscisse du point $\mathrm{B}\left(103^{\circ} \mathrm{C}\right)$ alors que l'abscisse du point $\mathrm{D}$ donne la température couplée à la température de régénération. Dans ce cas l'adsorption peut commencer à $T_{\mathrm{s}}^{\prime}=80^{\circ} \mathrm{C}$, et si la température du sorbant est maintenue à $30^{\circ} \mathrm{C}$ nous aurons donc production d'entropie irréversible comme nous l'avons indiqué dans le paragraphe précédent.

La quantité d'eau cyclée est donnée par la différence des teneurs en eau de la zéolithe soit $50 \mathrm{~g} / \mathrm{kg}$ de zéolithe pour le cycle $A B C D$ ou $78 \mathrm{~g}$ pour le cycle $\mathrm{A}^{\prime} \mathrm{B}^{\prime} \mathrm{CD}$.

D'après la figure 5 , nous voyons que $T_{\mathrm{s}}$ dépend de la température de condensation et de la température d'adsorption.
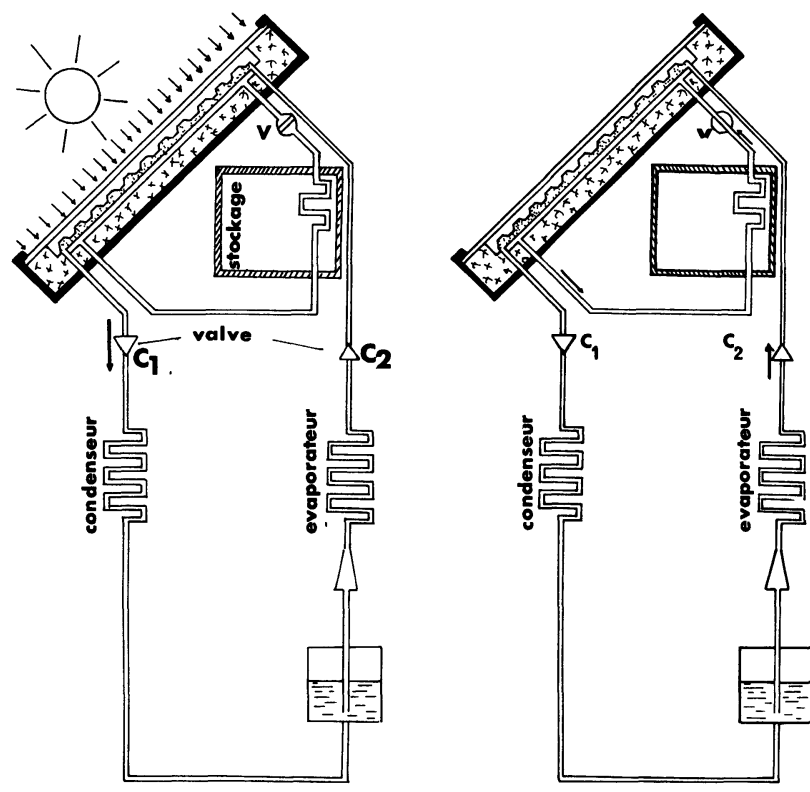

Fig. 6. - Principe de fonctionnement. a) Régénération : le clape $C_{2}$ est fermé et le clapet $C_{1}$ s ouvre dès que la température de la zéolithe atteint la valeur $T_{\mathrm{s}}$ et la condensation se produit. La vanne est fermée. h) Production de froid : Le clapet $C_{1}$ se ferme dès que le capteur se refroidit, le clapet $C_{2}$ s ouvre dès que la température atteint la valeur $T_{s^{\prime}}$ et l'évaporation s'effectue La vanne $\mathrm{V}$ est ouverte pour permettre la production d'eau chaude

[Running of the machine. a) Regeneration : the valve $C_{2}$ is closed and the valve $C_{1}$ opens as soon as the temperature in the zeolithe reaches the value $T_{s}$, the condensation then starts. The valve $\mathrm{V}$ is closed. h) Cooling phase : the valve $\mathrm{C}_{1}$ closes as soon as the collector cools down the valve $C_{2}$ opens when the temperature in the zeolithe reaches the value $T_{\mathrm{s}^{\prime}}$ and the evaporation starts The valve $\mathrm{V}$ is open to allow for the hot water production ]
3.2 FONCTIONNEMENT DE LA MACHINE [4]. - La machine consiste en un capteur plan à surface sélective (chrome noir) jouant alternativement le rôle d'adsorbeur et de générateur relié à un condenseur et un évaporateur avec des clapets antiretour (Fig. 6)

3.2.1 Description du capteur. - Le capteur que nous utilisons est composé de deux compartiments indépendants (Fig. 7). L'un des deux compartiments est rempli de zéolithe $13 \mathrm{X}$ et fait partie du circuit de réfrigération : il doit être étanche. La masse de zéolithe à l'intérieur de ce capteur dépend du site (pour l'instant nous fonctionnons avec $20 \mathrm{~kg}$ par $\mathrm{m}^{2}$ ). La zéolithe est simplement distribuée dans le collecteur avec des entretoises destinées à assurer la rigidité et le transfert thermique. La face avant de ce capteur a donc été traitée au chrome noir préalablement au remplissage. L'autre compartiment du capteur est simplement destiné à assurer la circulation d'un fluide caloporteur afin de produire de l'eau chaude sanitaire.

3.2.2 Phase de régénération. - A la fin de l'adsorption (au moment du lever du soleil) le capteur est à une température $T_{\text {ads }}$ et sous une pression de vapeur $p_{\mathrm{ev}}$ correspondant à la pression de vapeur dans l'évaporateur, c'est le point $\mathrm{A}$ du cycle de la figure 5. Le soleil chauffe le capteur dont la température s'élève, entraînant une désorption de la zéolithe et donc une élévation de pression dans le capteur qui va fermer le clapet antiretour C2 (Fig. 6a). Tant que la pression dans le capteur reste inférieure à la pression du condenseur, le clapet antiretour $\mathrm{Cl}$ reste fermé. Dès que la température atteint la valeur de la température seuil $T_{\mathrm{s}}$ (point B de la figure 5), le clapet $\mathrm{C} 1$ s'ouvre et la condensation s'amorce. Cette condensation se poursuivra jusqu'à ce que le capteur ait atteint sa température maximale (point $\mathrm{C}$ de la figure 5)

Tout au long de cette phase, la circulation du fluide caloporteur du second compartiment a été stoppée par la fermeture de la vanne $\mathrm{V}$.

3.2.3 Phase de production de froid. - Dès que la température dans le capteur baisse, le clapet $\mathrm{Cl}$ se ferme. On doit alors ouvrir la vanne V, ce qui va mettre le fluide caloporteur en circulation et va donc entraîner une rapide chute de température dans le capteur. Dès

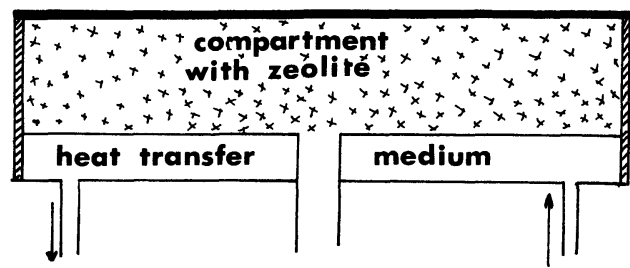

Fig. 7. - Coupe du capteur à deux compartiments que nous utilisons : - un compartiment rempli de zéolithe est relié au circuit de réfrigération, - le second compartiment assure la circulation du fluide caloporteur.

[Cross section of the collector consisting of two independent compartments : - one filled with zeolithe, - the other circulated by the heat transfer medium.] 
que la température dans le capteur est inférieure à la valeur $T_{\mathrm{s}}$, correspondant au point $\mathrm{D}$ de la figure 5 , le clapet $\mathrm{C} 2$ s'ouvre et l'évaporation peut commencer. L'évaporation se poursuivra toute la nuit et la température du capteur atteindra sa valeur minimale $T_{\text {ads }}$ le matin. Ce type de capteur à double enceinte a été conçu pour la climatisation : production de froid (et d'eau chaude sanitaire) en été et production de l'eau chaude pour le chauffage en hiver [4] : il suffit en hiver de stopper le circuit de réfrigération et l'on a alors un capteur plan ordinaire avec une plus grande inertie.

3.3 Modélisation. - Pour effectuer la modélisation d'un cycle, nous avons besoin de posséder les données suivantes :

- réseau d'isothermes ou bien isostères ou isobares du couple utilisé,

- enthalpies d'adsorption,

- les températures de fonctionnement (températures de l'évaporateur et de l'adsorbant à la fin de la sorption ainsi que la température du condenseur), - caractéristiques physiques du sorbant et du fluide,

- caractéristiques de la machine (masse des différents composants, etc...).

Un programme de calcul écrit en langage conversationnal APL et mis en mémoire au CIRCE à Orsay permet, avec les données mentionnées plus haut, de calculer sur IBM 370 :

- la température seuil $T_{\mathrm{s}}$,

- la puissance frigorifique ainsi que la chaleur fournie au cours de la régénération et donc le $C O P$ en fonction de la température de régénération.

Nous calculons le $C O P$ thermique et le $C O P$ solaire. Nous introduisons en fait trois $C O P$ thermiques :

$$
C_{0}=\frac{L\left(T_{\mathrm{ev}}\right)}{\Delta H\left(T_{\mathrm{reg}}\right)}
$$

est le rendement thermodynamique idéal correspondant aux quatre températures $\left(T_{\text {ev }}, T_{\text {ads }}, T_{\text {cond }}, T_{\text {reg }}\right)$ du cycle. $C_{0}$ est la limite vers laquelle tendrait une machine fonctionnant avec un cycle continu et avec une récupération de chaleur sensible idéale.

$C_{1}$ est le $C O P$ thermique qui prend en compte les chaleurs sensibles du sorbant et du sorbat. $C_{1}$ est la limite du cycle intermittent sans récupération de chaleur.

$C_{2}$ tient compte de la chaleur sensible de la machine. C'est le COP thermique réel d'une machine fonctionnant avec un cycle intermittent sans récupération de chaleur.

Pour calculer $C_{1}$ et $C_{2}$ nous avons introduit :

$Q_{1}$ : quantité de chaleur nécessaire à refroidir le sorbat de $T_{\text {cond }}$ à $T_{\text {ev }}$,

$Q_{\text {s }}$ : quantité de chaleur nécessaire pour porter la masse du générateur (sorbant et capteur) de $T_{\text {ads }}$ à $T_{\mathrm{s}}$,
$Q_{n}$ : quantité de chaleur sensible nécessaire pour porter la masse du générateur de $T_{n-1}$ à $T_{n}$.

$Q_{\text {s }}$ et $Q_{n}$ sont la somme de trois termes correspondant à :

- la capacité calorifique du capteur,

- la capacité calorifique du sorbant,

- la capacité calorifique du sorbat en phase adsorbée (la masse $M_{n}$ du sorbat étant une fonction décroissante de la température, $M_{0}$ étant la masse maximale en fin d'adsorption).

A la température de régénération $T_{N}$, le $C O P$ est donné par :

$$
C O P=\frac{L\left(T_{\mathrm{ev}}\right)\left(M_{0}-M_{N}\right)-Q_{1}}{\sum_{n=1}^{N} \Delta H\left(T_{n}\right) \times\left(M_{n-1}-M_{n}\right)+Q_{\mathrm{s}}+\sum_{n=1}^{N} Q_{n}}
$$

$C_{1}$ est le $C O P$ calculé avec une masse de capteur nul, pour $C_{2}$ nous avons pris une masse de capteur égale à la masse d'adsorbant.

Le $C O P$ solaire est calculé en prenant le rendement du capteur solaire dans chaque intervalle de température $T_{n-1}-T_{n}$.

3.4 DÉTERMINATION ET REPRÉSENTATION DES ISOTHERMES ET DES ENTHALPIES D'ADSORPTION. - Les données de base sont les isothermes et les enthalpies d'adsorption. Elles ont été déterminées expérimentalement [3] par des mesures de thermogravimétrie et de microcalorimétrie. La représentation analytique des résultats expérimentaux nous a posé des problèmes directement reliés aux théories de l'adsorption solide utilisées pour les corps microporeux. Rappelons brièvement qu'il existe deux types d'approche pour décrire l'adsorption sur zéolithes.

3.4.1 Représentation de type Langmuir [5]. L'équation d'une isotherme de type Langmuir est de la forme :

$$
K_{1}=\frac{P(1-\theta)}{0}
$$

où

$$
K_{1}=\exp \left(\frac{\Delta H}{R T}-\frac{\Delta S_{\mathrm{th}}^{0}}{R}\right)
$$

est indépendant de $\theta$ si $\Delta H$ et $\Delta S_{\text {th }}$ sont indépendants de $\theta . \Delta S_{\text {th }}^{0}$ est la partie thermique de l'entropie standard d'adsorption [6].

En fait $\Delta H$ est une fonction de $\theta$, différents auteurs ont calculé $\Delta H(\theta)$ et proposé des équations de Langmuir généralisées pour la représentation des isothermes. Avec ce type d'approche on peut passer de l'enthalpie d'adsorption à l'isotherme dès lors que l'on a une bonne représentation pour l'entropie de configuration (qui dans le cas sans interaction est égale à $R \ln \frac{1-\theta}{0}$. 
3.4.2 Théorie du potentiel d'adsorption [7]. - Cette approche semi-empirique propose une représentation des isothermes ne dépendant que de deux paramètres de la forme

$$
\theta=\frac{m}{m_{0}(T)}=\exp -D\left(T \log \frac{p_{0}}{p}\right)^{2}
$$

où

$-p_{0}$ est la pression de vapeur saturante du liquide pur,

- $m_{0}(T)$ est la masse maximale adsorbée,

$-D$ est un coefficient dépendant du couple utilisé et déterminé expérimentalement.

La chaleur isostérique est alors obtenue [7] simplement par dérivation en utilisant la formule de Clapeyron

$$
q_{\mathrm{st}}=L+R T \log \frac{p_{0}}{p}+\frac{\alpha R}{2 D}\left(\log \frac{p_{0}}{p}\right)^{-1}
$$

où

$-\alpha$ représente le coefficient d'expansion thermique du liquide.

3.4.3 Modélisation de l'adsorption sur zéolithes. La représentation de Dubinin rend très bien compte des isothermes d'adsorption de l'eau et du méthanol sur la zéolithe [3]. Néanmoins la formule (8) ne s'applique plus dès que l'on approche de $\theta=1$. L'entropie de configuration diverge en effet quand $\theta \rightarrow 1$ si l'on ne tient pas compte des interactions latérales.

Nous avons donc utilisé dans notre programme, la représentation de Dubinin pour les isothermes mais nous avons utilisé les résultats expérimentaux de Dijon [3] pour les enthalpies d'adsorption.

3.5 CRITÈres DE CHOIX DE COUPLES ET ÉCHelle EN TEMPÉRATURE. - Avant de présenter les résultats $\mathrm{du}$ programme de calcul, indiquons quels sont les critères pour le choix du couple le plus approprié à une application déterminée (caractérisée par des niveaux de température).

Les physico-chimistes caractérisent un couple sorbant sorbat par une isotherme (Fig. 8) donnant la masse de fluide adsorbée en fonction de la pression relative $p / p_{0}$ où $p_{0}$ est la pression de vapeur saturante du liquide à la température de l'adsorption.

Montrons que cette échelle $p / p_{0}$ est équivalente à une échelle en température. Considérons en effet un adsorbant solide à une température $T_{1}$ et un liquide à une température $T_{2}$ à la même pression de vapeur $p$. Les deux interfaces (liquide-vapeur à $T_{2}$ et solide-vapeur à $T_{1}$ ) sont décrits par une équation de Clausius-Clapeyron s'ils sont à l'équilibre. On a donc

$$
\begin{aligned}
& \log p=-\frac{\Delta H}{R T_{1}}+\frac{\Delta S_{\mathrm{s}}^{0}}{R} \\
& \log p=-\frac{L}{R T_{2}}+\frac{\Delta S^{0}}{R} .
\end{aligned}
$$

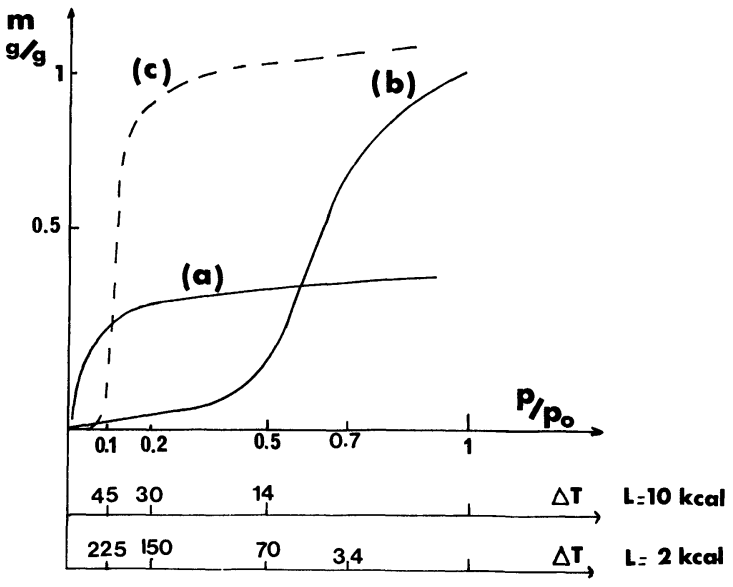

Fig. 8. - Isothermes dadsorption typiques En abscisse sont portées : - la pression relative, - le $\Delta T$ correspondant pour $L=10 \mathrm{kcal} / \mathrm{mole}$ (eau) et $2 \mathrm{kcal} / \mathrm{mole}$ (fréon). L'isotherme a est caractéristique des adsorbants microporeux (zéolithes, certains charbons). L'isotherme $\mathrm{b}$ est caractéristique des gels de silice et de certains charbons. $L$ isotherme $c$ représente l'isotherme idéal pour la réfrigération avec l'eau comme fluide.

[Typical isotherms of adsorption : adsorbed mass versus : - the relative pressure, - or the equivalent $\Delta T$ calculated for $L=10 \mathrm{kcal} /$ mole (water) and $L=2 \mathrm{kcal} / \mathrm{mole}$ (freon). a, is a typical isotherm of microporous adsorbents (zeolithes, some carbons). b, is a typical isotherm of silica gels and other carbons. c, would be the ideal isotherm for cooling when water is the refrigerant fluid.]

Par ailleurs la pression de vapeur saturante $p_{0}$ est donnée par

$$
\log p_{0}=-\frac{L}{R T_{1}}+\frac{\Delta S^{0}}{R} .
$$

On a donc simplement

$$
\log \frac{p}{p_{0}}=-\frac{L}{R} \frac{\Delta T}{T_{1} T_{2}}
$$

avec $\Delta T=T_{1}-T_{2}$

Chaque point de l'isotherme donne donc la masse adsorbée en fonction de la différence de température entre le solide et le liquide. Au cours d'un cycle (Fig. 9), on cherche à adsorber le maximum de fluide lorsque $\Delta T=T_{\mathrm{amb}}-T_{\mathrm{ev}}$ et à adsorber le minimum lorsque $\Delta T=T_{\mathrm{reg}}-T_{\mathrm{amb}}$. Or un résultat phénoménologique important est qu'une isotherme représentée en $p / p_{0}$ est quasi indépendante de la température. L'isotherme idéale sera donc une isotherme du type $\mathrm{C}$ sur la figure 8 pour laquelle la marche sera située à la pression relative correspondant au bon écart de température $\Delta T=T_{\mathrm{amb}}-T_{\mathrm{ev}}$. Notons que d'après la formule (12) la position de la marche dépend de la valeur de la chaleur latente, elle sera donc très différente suivant que l'on utilisera l'eau, l'ammoniac ou un fréon.

Sur la figure 8, nous avons présenté trois isothermes correspondant à l'adsorption de l'eau :

- l'isotherme a caractéristique des absorbants microporeux permet de travailler à grand écart de température $\Delta T=T_{\mathrm{amb}}-T_{\mathrm{ev}}$ mais exige corrélative- 

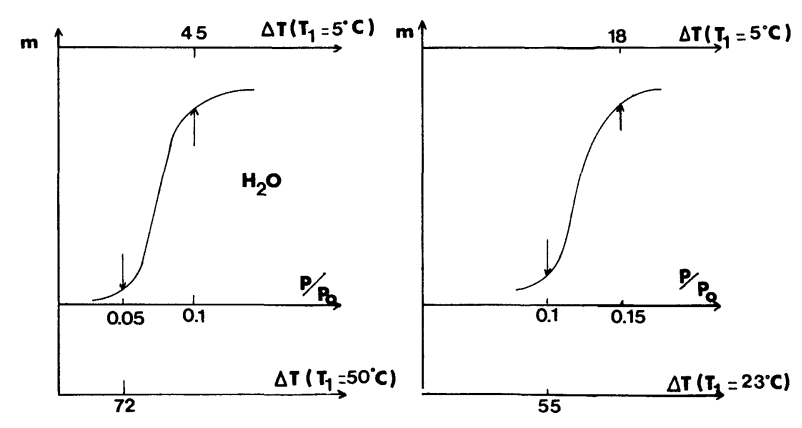

$$
\text { a } \begin{aligned}
T_{\text {ev }} & =5^{\circ} \mathrm{C} \\
T_{\text {ads }} & =T_{\text {cond }}=50^{\circ} \mathrm{C} \\
T_{\text {reg }} & =122 \mathrm{C}
\end{aligned}
$$

Fig. 9 - Représentation de cycles sur une isotherme

a) Pour un couple présentant une marche d'adsorption comprise entre $P / P_{0}=0,05$ et $P P_{0}=0,1$ avec l'eau comme fluide réfrigérant on obtient au moment de l'adsorption $\Delta T=45^{\circ} \mathrm{C}$ et au moment de la régénération $\Delta T=72^{\circ} \mathrm{C}$ ce qui permet de fonctionner avec les températures : $T_{\mathrm{ev}}=5^{\circ} \mathrm{C}, T_{\mathrm{ads}}=T_{\text {cond }}=50^{\circ} \mathrm{C}$ et $T_{\text {reg }}=122^{\circ} \mathrm{C}$.

b) Pour un couple présentant une marche d'adsorption comprise entre $P / P_{0}=0,1$ et $P / P_{0}=0,15$, les températures de fonctionnement seront : $T_{\mathrm{ev}}=5^{\circ} \mathrm{C}, T_{\text {ads }}=T_{\text {cond }}=23^{\circ} \mathrm{C}$ et $T_{\text {reg }}=78^{\circ} \mathrm{C}$. [Representation of two cycles on two isotherms $a$ ) If the step of adsorption of the pair is between $P / P_{0}=0.05$ and $P / P_{0}=0.1$ with water as refrigerant fluid, that leads to $\Delta T=45^{\circ} \mathrm{C}$ for the adsorption and to $\Delta T=72^{\circ} \mathrm{C}$ for the regeneration. The working temperatures are then $T_{\mathrm{ev}}=5^{\circ} \mathrm{C}, T_{\mathrm{ads}}=T_{\text {cond }}=50^{\circ} \mathrm{C}$ and $T_{\text {reg }}=122{ }^{\circ} \mathrm{C}$.

h) If the step is between $P / P_{0}=0.1$ and $P / P_{0}=0.15$ that leads to : $T_{\text {ev }}=5^{\circ} \mathrm{C}, T_{\text {ads }}=T_{\text {cond }}=23^{\circ} \mathrm{C}$ and $\left.T_{\text {reg }}=78^{\circ} \mathrm{C}\right]$

ment de travailler à fort écart de température au moment de la régénération,

- l'isotherme $b$ en revanche caractéristique de certains gels et charbons ne permet de travailler qu'à très faible écart de température $\Delta T=T_{\mathrm{amb}}-T_{\mathrm{ev}_{\mathrm{v}}}$ mais permet d'utiliser des basses températures pour la régénération,

- l'isotherme c serait l'isotherme idéale pour la réfrigération solaire. Il nous semble qu'une telle isotherme pourrait être obtenue avec certains charbons actifs.

4. Résultats. - Sur la figure 10 , nous présentons les résultats typiques que nous permet d'obtenir le programme de calcul. Nous présentons donc le rendement de Carnot correspondant à une machine tritherme fonctionnant avec les températures $T_{\mathrm{ev}}=0^{\circ}$, $T_{0}=30^{\circ} \mathrm{C}$ et $T_{\text {reg }}$ variable.

$C_{0}$ est le rendement thermodynamique idéal de la machine quadritherme utilisée dans laquelle $T_{\mathrm{ev}}=0^{\circ} \mathrm{C}, T_{\text {cond }}=30^{\circ} \mathrm{C}$ et $T_{\text {ads }}$ est une fonction de $T_{\text {reg }}$ (cf. annexe) mais la valeur indiquée $T_{\text {ads }}=30^{\circ} \mathrm{C}$ correspond à la température minimale d'adsorption du cycle intermittent.

$C_{2}$ est le $C O P$ correspondant à une masse métallique égale à la masse d'adsorbant. Les $C O P$ solaires ont été calculés avec des courbes réponses de capteurs

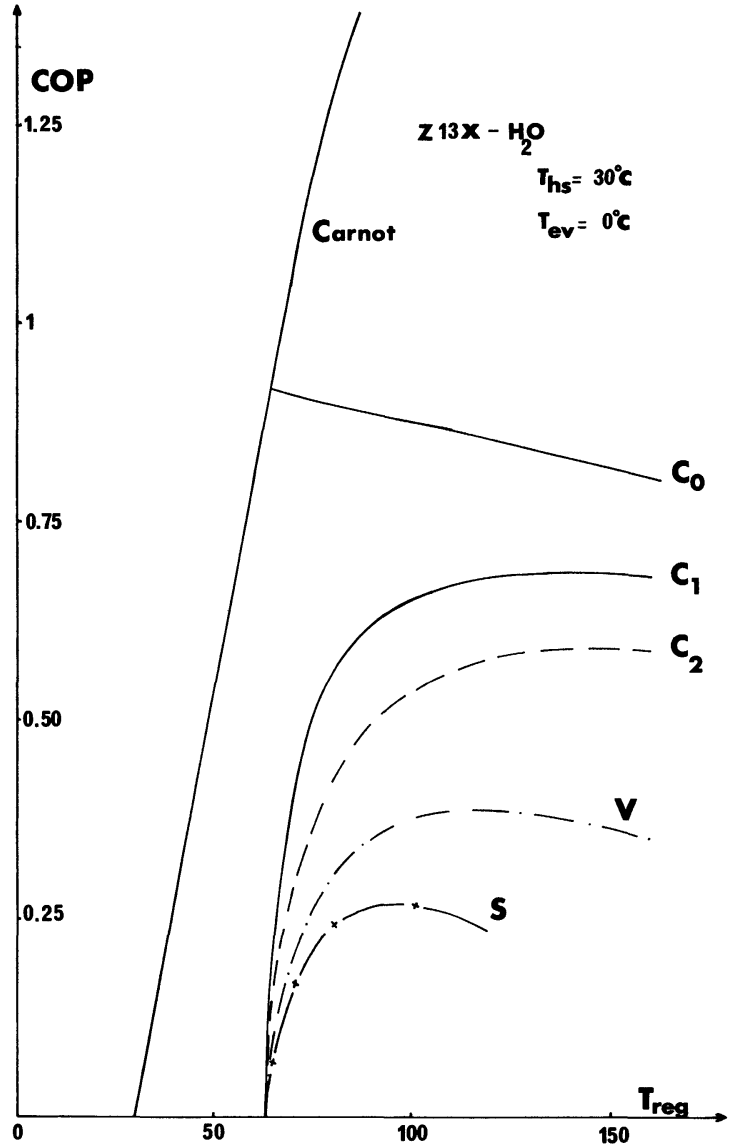

Fig. 10. - Présentation des $C O P$ en fonction de la température de régénération pour le couple zéolithe $13 \mathrm{X}-\mathrm{H}_{2} \mathrm{O}$ avec $T_{\mathrm{ev}}=0^{\circ} \mathrm{C}$, $T_{\text {ads }}=T_{\text {cond }}=30^{\circ} \mathrm{C}$. COP de Carnot ; $C_{0}, C_{1}, C_{2}$ sont les $C O P$ thermiques définis dans le texte; $V$ et $S$ sont les $C O P$ solaires.

[COP versus the regenerating temperature for the zeolithe $13 \mathrm{X}-\mathrm{H}_{2} \mathrm{O}$ pair with $T_{\mathrm{ev}}=0^{\circ} \mathrm{C}, T_{\mathrm{ads}}=T_{\text {cond }}=30^{\circ} \mathrm{C}$. Carnot $C O P ; C_{0}, C_{1}$ and $C_{2}$ are the thermal $C O P$ as defined in the text $V$ and $S$ are solar $C O P$.]

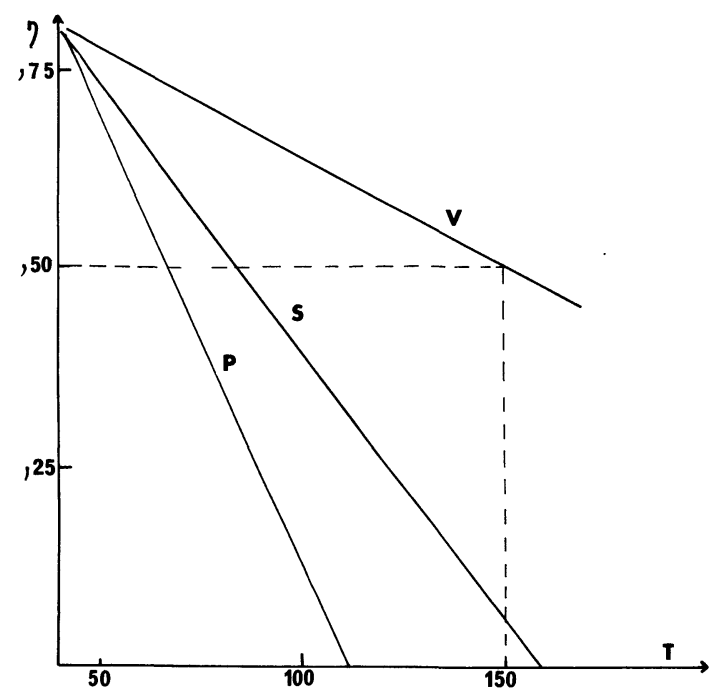

Fig. 11. - Courbes typiques de rendement de capteur retenues pour nos calculs : $\mathrm{P}$ capteur plan simple, $\mathrm{S}$ capteur plan à surface sélective, $\mathrm{V}$ capteur sous vide

[Typical curves of performance of collectors taken for our calculations : P : flat plate collector, $\mathbf{S}$ : flat plate collector, with selective surface, $\mathrm{V}$ : evacuated collector ] 
présentées sur la figure 11 ( $\mathrm{P}$ : plan simple, $\mathrm{S}$ : plan avec surface sélective, $\mathrm{V}$ : capteur sous vide).

4.1 COMPARAISON DE DIFFÉRENTS SORBANTS. Sur les figures 12 et 13 , nous présentons une comparaison des calculs des performances (nous présentons le COP $C_{1}$ ) de cycles utilisant l'eau comme fluide réfrigérant et quatre sorbants différents : $\mathrm{LiBr}$, alumine activée, zéolithe $13 \mathrm{X}$ et zéolithe $4 \mathrm{~A}$. Pour $\mathrm{LiBr}$ nous avons utilisé les données physicochimiques rassemblées dans l'ASHRAE handbook of Fundamentals [8], pour l'alumine activée les données que nous a fournies Rhône-Poulenc [9] et pour les zéolithes les données de Mme Simonot [10] à Dijon.

Sur la figure 14, nous présentons le rapport des $\operatorname{COP}\left(C_{0}\right.$ et $\left.C_{2}\right)$ au $C O P$ de Carnot pour la zéolithe $13 \mathrm{X}$ et le $\mathrm{LiBr}$.

Sur la figure 15 , nous présentons le maximum du rapport $C_{2} /$ Carnot pour trois sorbants $(\mathrm{LiBr}$, alumine activée et zéolithe $13 \mathrm{X}$ ) en fonction de la différence de température entre la source froide et l'évaporateur (la température de l'évaporateur a été prise égale à $5{ }^{\circ} \mathrm{C}$ pour calculer les courbes de la figure 15).

De l'analyse de ces courbes nous tirons les conclusions suivantes :

- il n'y a pas de sorbant idéal pour toutes les gammes de température,

- le $\mathrm{LiBr}$ et l'alumine activée présentent leur efficacité maximale à faible différence de température entre la source froide et l'évaporateur,

- la zéolithe $13 \mathrm{X}$ présente au contraire son efficacité maximale pour une différence de température de $50^{\circ} \mathrm{C}$ entre la température de la source froide et la température de l'évaporateur,

- les couples LiBr-eau et Alumine activée-eau sont donc très bien adaptés pour la climatisation avec refroidissement à eau de la source froide,

- le couple zéolithe $13 \mathrm{X}$-eau est bien adapté pour la climatisation avec refroidissement à air de la source froide (Fig. 18).

4.2 Comparaison DE Différents Fluides. - En changeant de fluide, on modifie l'interaction sorbantsorbat ce qui a pour conséquence de modifier $U_{0}$ (cf. § 2) et donc de déplacer le domaine de température dans lequel le couple a son efficacité optimale. Une étude comparative des propriétés d'adsorption de l'eau, du méthanol et de l'éthanol a été faite récemment à Dijon [11]. L'interaction sorbant-sorbat va en croissant dans l'ordre : eau-méthanol-éthanol. Ceci a trois conséquences :

- les températures seuil de l'éthanol et du méthanol sont plus faibles que celle correspondant à l'eau,

- les $C O P$ avec le méthanol et l'éthanol sont plus faibles que celui du cycle fonctionnant avec l'eau,

- juste au-dessus de la température seuil le rapport $C_{2} /$ Carnot est relativement élevé avec le méthanol et l'éthanol.
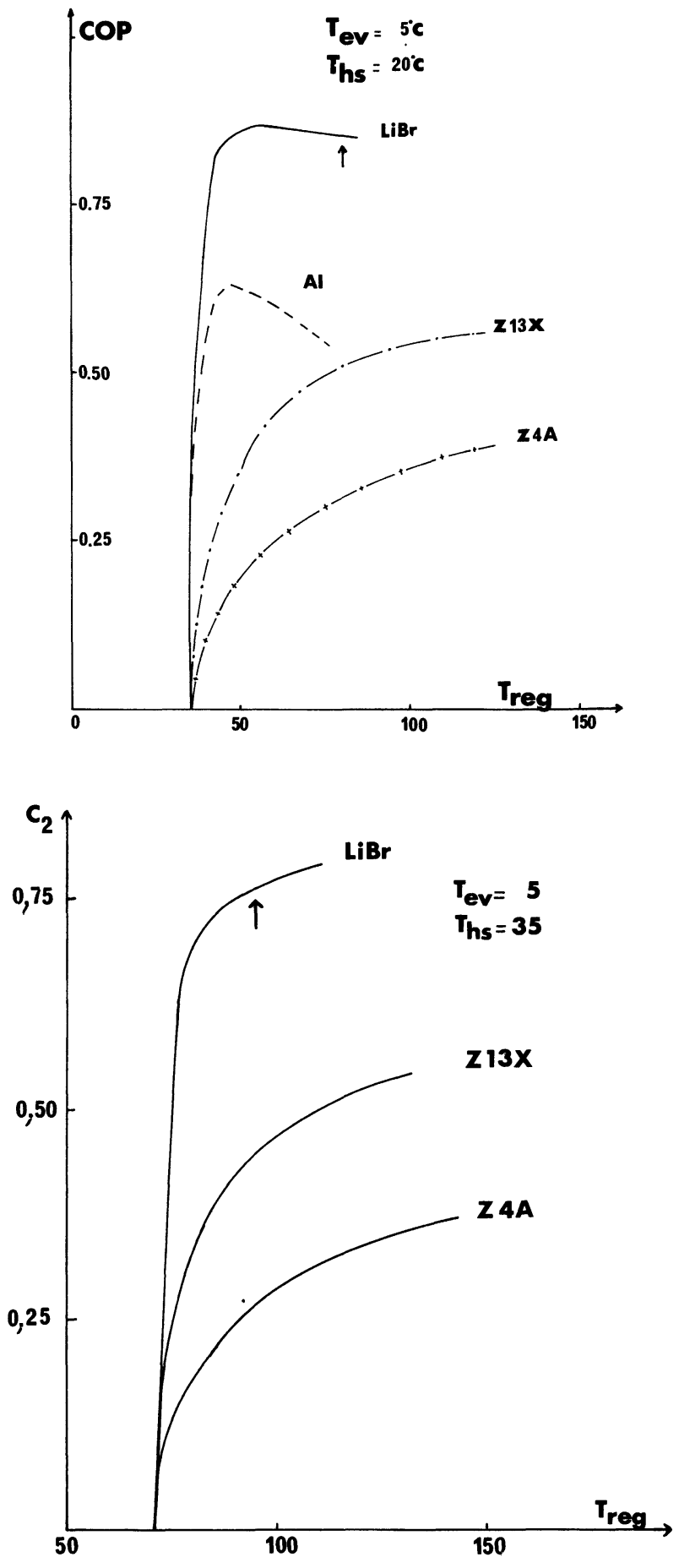

Figs. 12 et 13. - Comparaisons des $C O P$ thermiques $\left(C_{1}\right)$ de différents couples utilisant l'eau comme fluide réfrigérant. $T_{\mathrm{ev}}=5^{\circ} \mathrm{C}$ et la température de la source froide est de $20^{\circ} \mathrm{C}$ sur la figure 12 et $35^{\circ} \mathrm{C}$ sur la figure 13 . La flèche représente la limite de cristallisation pour le couple $\mathrm{LiB}_{2}-\mathrm{H}_{2} \mathrm{O}$.

[Comparison of thermal $\operatorname{COP}\left(C_{1}\right)$ for various pairs working with $\mathrm{H}_{2} \mathrm{O}$ as refrigerant fluid. $T_{\mathrm{ev}}=5^{\circ} \mathrm{C}$ and the heat sink temperature is $20^{\circ} \mathrm{C}$ on the figure 12 and $35^{\circ} \mathrm{C}$ on the figure 13 . The arrow represents the cristallization limit for the $\mathrm{LiBr}-\mathrm{H}_{2} \mathrm{O}$ pair.]

Nous présentons sur la figure 16 les résultats caractéristiques pour le méthanol avec une température d'évaporateur de $-10^{\circ} \mathrm{C}$. 

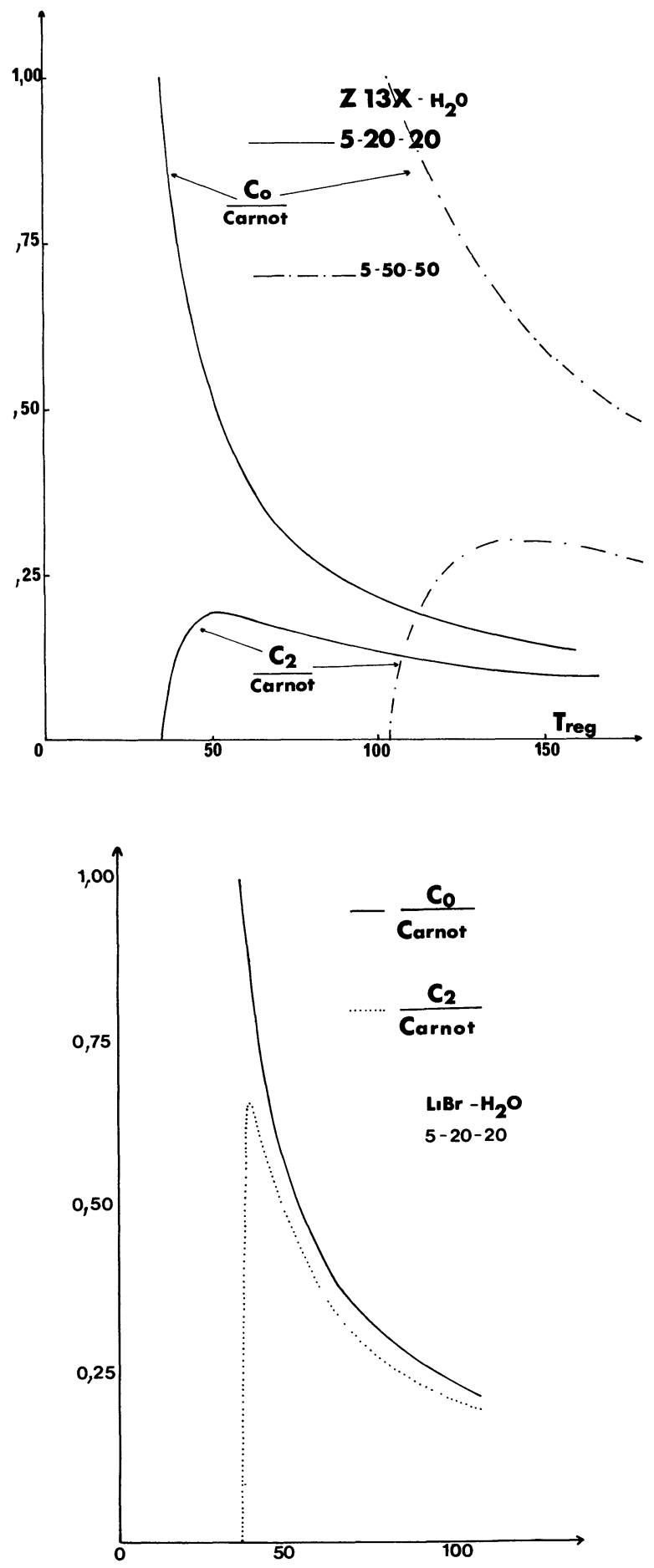

Fig. 14. - Courbes calculées de $C_{0} /$ Carnot et $C_{2} /$ Carnot pour les couples zéolithe $13 \mathrm{X}-\mathrm{H}_{2} \mathrm{O}$ et $\mathrm{LiBr}-\mathrm{H}_{2} \mathrm{O} \cdot T_{\mathrm{ev}}=5^{\circ} \mathrm{C}$ et température de la source froide $20^{\circ} \mathrm{C}$ et $50^{\circ} \mathrm{C}$

[Variation of $C_{0} /$ Carnot and $C_{2} /$ Carnot versus the regenerating temperature for the pairs zeolithe $13 \mathrm{X}-\mathrm{H}_{2} \mathrm{O}$ and $\mathrm{LiBr}-\mathrm{H}_{2} \mathrm{O}$. $T_{\mathrm{ev}}=5^{\circ} \mathrm{C}$ and heat sink temperature of $20^{\circ} \mathrm{C}$ and $50^{\circ} \mathrm{C}$.]

4.3 INFLUENCE D'UNE VARIATION DES TEMPÉRATURES DE FONCTIONNEMENT. - Nous avons déjà présenté sur les figures 12,13 et 15 l'influence d'une modification de la température de la source froide.

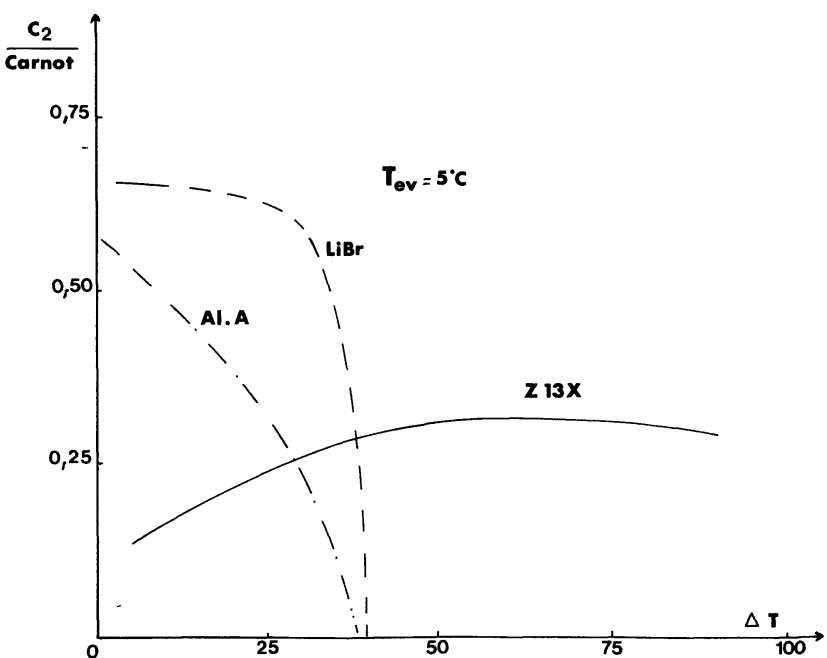

Fig. 15. - Variation du maximum de $C_{2} /$ Carnot déterminé sur la courbe de la figure 14 en fonction de la différence de température entre source froide et température de l'évaporateur pour trois couples : - $\mathrm{LiBr}-\mathrm{H}_{2} \mathrm{O}$; - Alumine activée- $\mathrm{H}_{2} \mathrm{O}$ : - Zéolithe $13 \mathrm{X}-\mathrm{H}_{2} \mathrm{O}$

[Variation of the maximum of the function $C_{2} /$ Carnot as determined on the figure 14 versus the temperature difference between the heat sink and the evaporator for three pairs : $-\mathrm{LiBr}-\mathrm{H}_{2} \mathrm{O}$; - Activated alumina- $\mathrm{H}_{2} \mathrm{O}$; - Zeolithe $13 \mathrm{X}-\mathrm{H}_{2} \mathrm{O}$.]

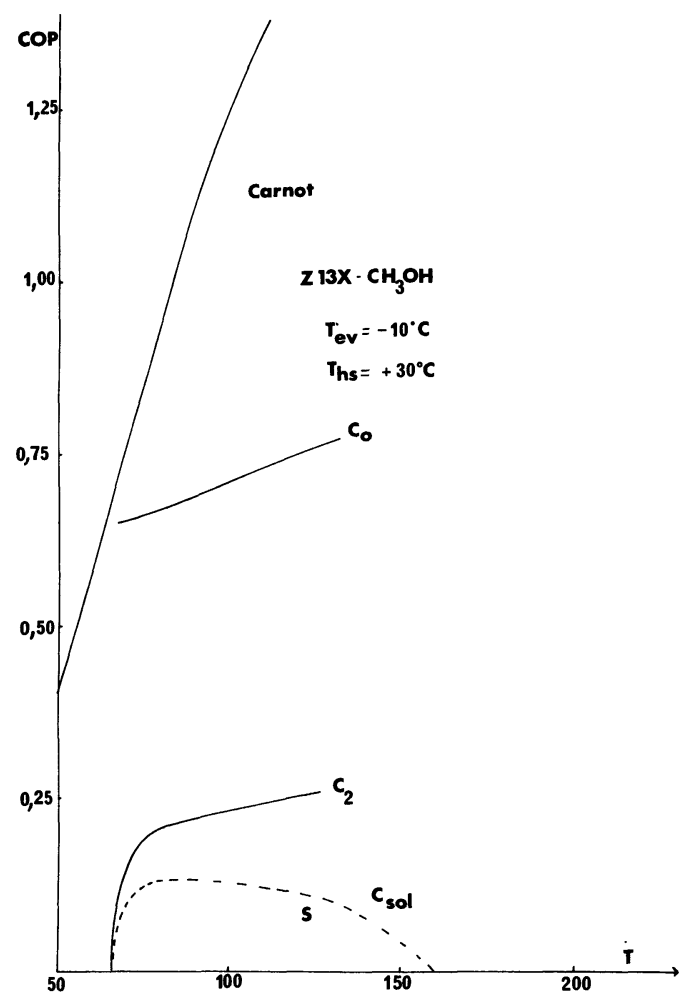

Fig. 16. - $C O P$ calculés pour le couple zéolithe $13 \mathrm{X}_{-}-\mathrm{CH}_{3} \mathrm{OH}$ pour $T_{\mathrm{ev}}=-10^{\circ} \mathrm{C}$ et température de la source froide $=30^{\circ} \mathrm{C}$.

[Calculated $\mathrm{COP}$ for the zeolithe $13 \mathrm{X}-\mathrm{CH}_{3} \mathrm{OH}$ pair for $T_{\mathrm{ev}}=-10^{\circ} \mathrm{C}$ and heat sink temperature of $30^{\circ} \mathrm{C}$.] 


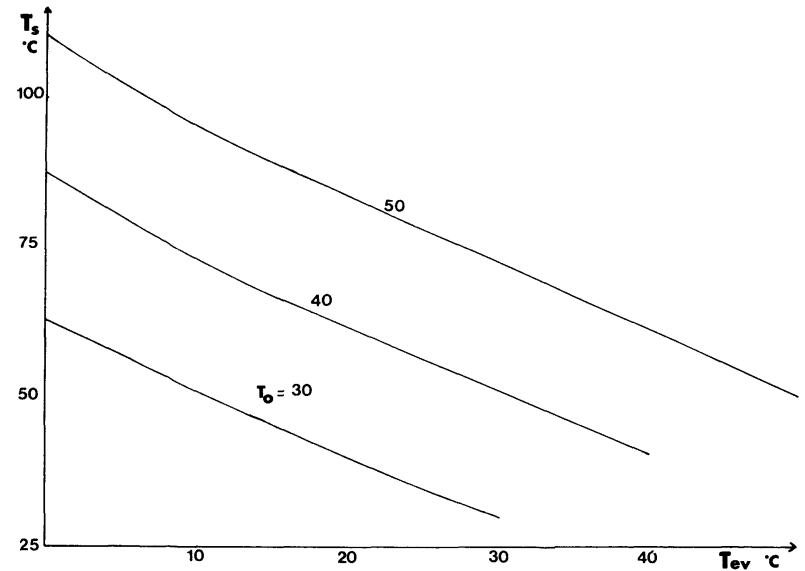

Fig. 17 - Température seuil en fonction de la température de l'évaporateur pour le couple zéolithe $13 \mathrm{X}-\mathrm{H}_{2} \mathrm{O}$ pour trois valeurs de la température de condensation $\left(30^{\circ}, 40\right.$ et $\left.50^{\circ} \mathrm{C}\right)$

[Variation of the threshold temperature $\mathrm{T}_{\mathrm{S}}$ versus the temperature of the evaporator for the zeolithe $13 \mathrm{X}-\mathrm{H}_{2} \mathrm{O}$ pair for three values of the condensing temperature $\left(30,40\right.$ and $\left.50^{\circ} \mathrm{C}\right)$.]

Sur la figure 17, nous présentons la variation de la température seuil $T_{\mathrm{s}}$ en fonction de la température d'évaporation $T_{\text {ev }}$ pour le cycle zéolithe $13 \mathrm{X}-\mathrm{H}_{2} \mathrm{O}$ et pour trois valeurs différentes de la source froide $\left(30^{\circ}, 40^{\circ}\right.$ et $\left.50^{\circ} \mathrm{C}\right)$.

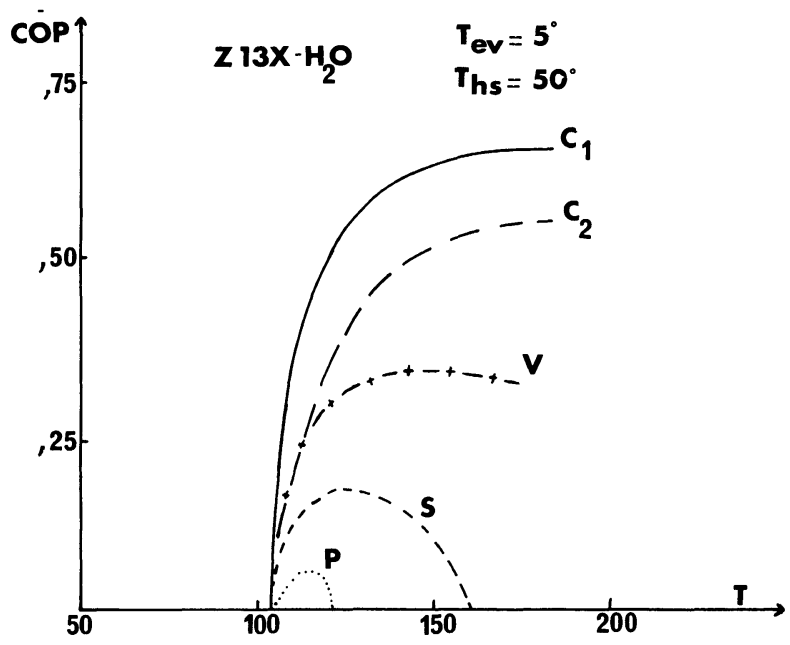

Fig. 18. - COP calculés pour le couple zéolithe $13 \mathrm{X}-\mathrm{H}_{2} \mathrm{O}$ avec $T_{\mathrm{ev}}=5^{\circ} \mathrm{C}$ et température de la source froide à $50{ }^{\circ} \mathrm{C}$.

[Calculated $C O P$ for the zeolithe $13 \mathrm{X}-\mathrm{H}_{2} \mathrm{O}$ pair with $T_{\mathrm{ev}}=5^{\circ} \mathrm{C}$ and the heat sink temperature of $50^{\circ} \mathrm{C}$.]

4. 4 CHOIX DU CAPTEUR APPROPRIÉ. - Figure 19, sont présentées les courbes de $C O P$ solaire correspondant à trois températures différentes de source froide $\left(20^{\circ} \mathrm{C}, 35^{\circ} \mathrm{C}\right.$ et $\left.50{ }^{\circ} \mathrm{C}\right)$ et les trois types de capteur de la figure 7. Figure 20, on présente les courbes de $C O P$ solaire pour le couple alumine activée-eau avec une température de source froide de $20^{\circ} \mathrm{C}$.

Nous voyons que pour une faible différence de
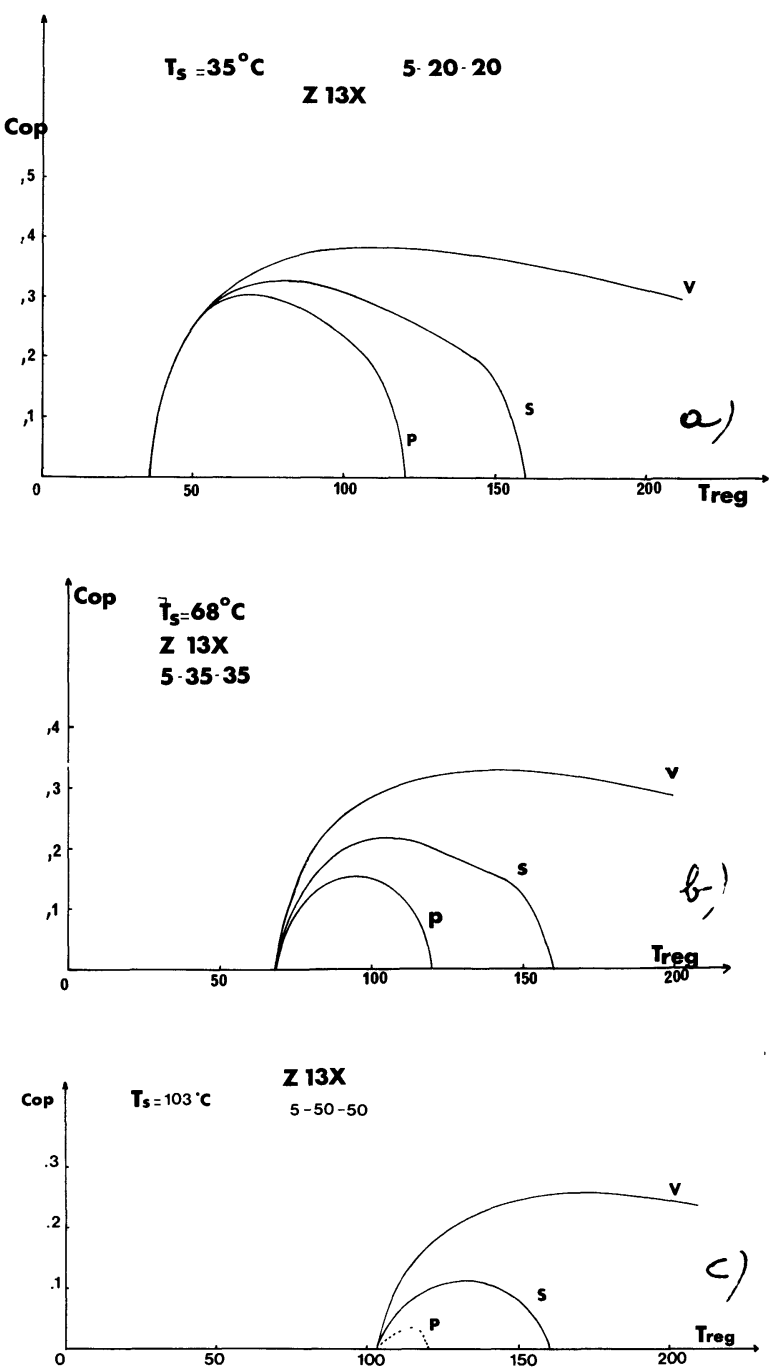

Fig. 19. - COP solaires calculés avec les trois types de capteurs de la figure 11 et trois températures différentes de la source froide pour le couple zeolithe $13 \mathrm{X}-\mathrm{H}_{2} \mathrm{O}$. a) Température de la source froide $=20^{\circ} \mathrm{C} ; b$ ) Température de la source froide $=35^{\circ} \mathrm{C}$; c) Température de la source froide $=50^{\circ} \mathrm{C}$.

[Calculated solar COP with the three types of collectors of figure 11 and three distinct temperatures of the heat sink for the pair zeolithe $13 \mathrm{X}-\mathrm{H}_{2} \mathrm{O}$. a) Heat sink temperature $=20^{\circ} \mathrm{C} ; b$ ) Heat sink temperature $\left.=35^{\circ} \mathrm{C} ; c\right)$ Heat sink temperature $=50^{\circ} \mathrm{C}$.]

température entre source froide et évaporateur $\left(\Delta T=15^{\circ} \mathrm{C}\right)$, le capteur plan simple suffit. En revanche, pour une forte différence de température $\left(\Delta T=45^{\circ} \mathrm{C}\right)$ il est indispensable d'utiliser un capteur performant à haute température (type capteur à surface sélective ou même mieux capteur sous vide).

5. Conclusion. - Cette étude nous a permis de montrer (et de réaliser un appareil de démonstration) que le cycle zéolithe $13 \mathrm{X}-\mathrm{H}_{2} \mathrm{O}$ était adapté pour la climatisation avec refroidissement à air de la source froide. Ce cycle peut fonctionner sans aucune énergie d'appoint et sans partie mobile. Néanmoins, il faut utiliser un capteur performant à des températures comprises entre 100 et $150^{\circ} \mathrm{C}$, avec la technologie 


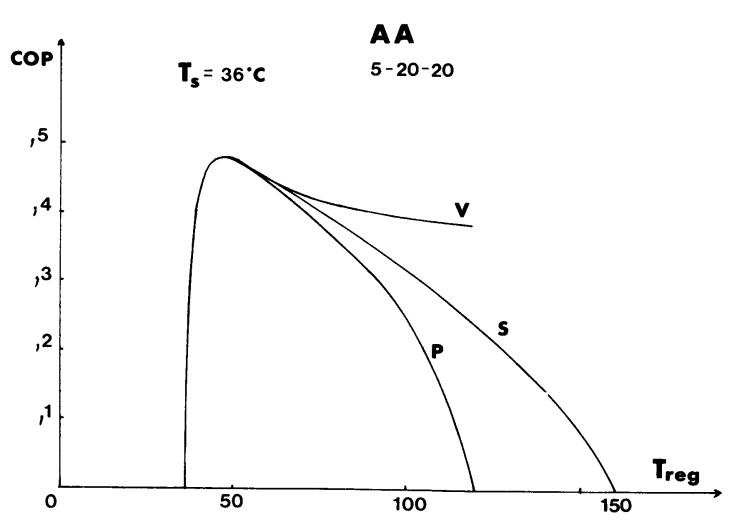

Fig. 20. - $C O P$ solaire calculé pour le couple alumine activée $\mathrm{H}_{2} \mathrm{O}$ avec les températures $T_{\mathrm{ev}}=5^{\circ} \mathrm{C}$ et $T_{\text {ads }}=T_{\text {cond }}=20^{\circ} \mathrm{C}$.

[Calculated solar $C O P$ for the activated alumina $\mathrm{H}_{2} \mathrm{O}$ pair with $T_{\mathrm{ev}}=5^{\circ} \mathrm{C}$ and heat sink temperature of $20^{\circ} \mathrm{C}$.]

existante ce sont les capteurs plans à surface sélective (chrome noir) les mieux adaptés. Il apparaît que si les capteurs sous vide se développent, ce seront eux qui s'imposeront mais alors la technologie sera vraisemblablement différente puisque l'adsorbant ne sera plus distribué à même le capteur. Des études sont en cours dans cette direction.

Annexe. - Considérons les transferts de chaleur associés à un transfert de masse d'une phase liquide vers une phase sorbée (liquide ou adsorbée) en passant par la phase vapeur (cf. Fig. 2).

Ce transfert peut se faire de la phase sorbée vers la phase liquide, c'est alors l'étape de régénération. Si au contraire le transfert s'effectue de la phase liquide vers la phase sorbée, c'est l'étape production de froid ; si aucun transfert de masse n'a lieu, le système se trouve dans son état stationnaire.

ETAPE DE RÉGÉNÉRATION. - Soient $\Delta H$ la chaleur latente de sorption et $L$ la chaleur latente de vaporisation du liquide pur. Appelons $U_{0}$ l'énergie interne produite et $\Delta Q$ la chaleur échangée avec le milieu extérieur sous une autre forme que chaleur latente.

Appliquant les deux premiers principes de la thermodynamique, on a

$$
\begin{gathered}
\Delta H-L=\Delta Q+U_{0} \\
\frac{L+\Delta Q}{\Delta H} \geqslant \frac{T_{\text {cond }}}{T_{\text {reg }}}
\end{gathered}
$$

(a.2) peut s'écrire

$$
\frac{\Delta H}{T_{\text {reg }}}-\frac{L}{T_{\text {cond }}} \leqslant \frac{\Delta Q}{T_{\text {cond }}} .
$$

Si le processus est réversible, l'égalité est satisfaite et de (a.1), on déduit bien sûr

$$
U_{0} \leqslant \Delta H\left(1-\frac{T_{\text {cond }}}{T_{\text {reg }}}\right) .
$$

ETAPE DE PRODUCTION DE FROID. - On obtient des équations équivalentes, mais bien entendu le sens des inégalités est inversé et les températures de fonctionnement sont maintenant $T_{\mathrm{ev}}$ et $T_{\text {sor }}$ où $T_{\text {sor }}$ est la température de sorption

$$
\frac{\Delta H}{T_{\text {sor }}}-\frac{L}{T_{\mathrm{ev}}} \geqslant \frac{\Delta Q}{T_{\mathrm{ev}}} .
$$

L'énergie interne qu'il a fallu fournir a été

$$
U_{0} \geqslant \Delta H\left(1-\frac{T_{\mathrm{ev}}}{T_{\text {sor }}}\right)
$$

l'égalité correspondant à un processus réversible. Or $\Delta H$ est une fonction de la température et d'une autre paramètre (concentration ou taux de remplissage) c'est à ce niveau qu'apparaît la différence entre machine tritherme et machine quadritherme. En effet, si l'énergie $U_{0}$ dont on dispose est grande car elle provient d'une température de régénération élevée, on ne pourra pas la diviser en deux ou trois dans l'étape de production de froid.

Etat STATIONNAIRE. - Dans l'état stationnaire, chaque interface est décrit par une équation de Clausius-Clapeyron. La combinaison de ces deux équations donne :

$$
\frac{\Delta H\left(C, T_{2}\right)}{T_{2}}-\frac{L\left(T_{1}\right)}{T_{1}}=\Delta S_{\mathrm{e}}
$$

où

$$
\Delta S_{\mathrm{e}}=\int_{T_{1}}^{T_{2}} \frac{C_{\mathrm{pg}}-C_{\mathrm{PL}}}{T} \mathrm{~d} T-\delta S\left(C, T_{2}\right)
$$

- $T_{1}$ est la température du liquide pur,

- $T_{2}$ est la température du sorbant,

- $\delta S$ est l'entropie différentielle entre la phase sorbée et le liquide pur à la même température $T_{2}$,

- $C_{\mathrm{pg}}$ est la chaleur spécifique de la vapeur,

- $C_{\mathrm{pL}}$ est la chaleur spécifique du liquide.

La comparaison des équations (a.3), (a.5) et (a.7) nous montre donc que la quantité $\Delta Q / T$ est simplement égale à $\Delta S_{\mathrm{e}}$ donné par l'équation (a.8).

SENS D'Évolution. - Le sens de l'évolution est donné par les inégalités (a.3) et (a.5). En effet si pour un couple de températures, le système est à l'état stationnaire, la condition (a.7) est satisfaite, toute modification d'une température va entraîner une évolution; ainsi si dans l'équation (a.7), $T_{2}$ augmente et prend la valeur $T_{2}+\delta T_{2}$, l'égalité n'est plus satisfaite et l'on a alors :

$$
\frac{\Delta H}{T_{2}+\delta T_{2}}-\frac{L}{T_{1}}=\Delta S<\Delta S_{\mathrm{e}}=\frac{\Delta Q}{T_{1}}
$$

ce qui, d'après (a.3) correspond à la régénération. 
En revanche si $T_{2}$ diminue et prend la valeur $T_{2}-\delta T_{2}$, on a alors :

$$
\frac{\Delta H}{T_{2}-\delta T_{2}}-\frac{L}{T_{1}}=\Delta S>\Delta S_{\mathrm{e}}=\frac{\Delta Q}{T_{1}}
$$

ce qui, d'après (a .5) correspond à la production de froid.

TEMPÉRATURE SEUIL. - La température seuil peut être calculée en utilisant la formule de ClausiusClapeyron.

Revenons à la figure 5 : lorsqu'on passe du point $\mathrm{A}$ au point $\mathrm{B}$, la pression change de $P_{\mathrm{ev}}$ à $P_{\text {cond }}$ et la température de $T_{\text {ads }}$ à $T_{\mathrm{s}}$. Or nous avons :

$$
\frac{\mathrm{d} \log P}{\mathrm{~d} T}=\frac{\Delta H(\theta, T)}{R T^{2}}
$$

où $\Delta H(\theta, T)$ est l'enthalpie d'adsorption (que nous prendrons indépendante de la température), $T$ est la température du sorbant et $P$ la pression de vapeur au-dessus du sorbant. L'intégration de la relation (a.11) le long de $\mathrm{AB}$ donne :

$$
\log \frac{P_{\text {cond }}}{P_{\text {ev }}}=\int_{T_{\text {ads }}}^{T 、} \frac{\Delta H(\theta, T)}{R T^{2}} \mathrm{~d} T .
$$

Du côté liquide nous avons de même :

$$
\frac{\mathrm{d} \log P}{\mathrm{~d} T}=\frac{L}{R T^{2}}
$$

où $T$ est la température du liquide et $P$ la pression au-dessus du liquide. Intégrant entre la température d'évaporateur et celle du condenseur on obtient :

$$
\log \frac{P_{\text {cond }}}{P_{\text {ev }}}=\int_{T_{\text {ev }}}^{T_{\text {cond }}} \frac{L}{R T^{2}} \mathrm{~d} T .
$$

( $\left.{ }^{1}\right)$ Nous utilisons $c$ et 0 car les deux notations existent, $c$ représente la concentration de sel dans une solution $\left(c=\frac{\text { masse de sel }}{\text { masse totale }}\right)$ alors que 0 représente un taux de sorption $\left(0=\frac{\text { masse de fluide }}{\text { masse de sorbant }}\right)$ il existe bien sûr une relation simple entre les deux $c=\frac{1}{1+\theta}$
La combinaison de (a.12) et (a.14) donne :

$$
\int_{T_{\mathrm{ev}}}^{T_{\mathrm{cond}}} L d\left(\frac{1}{T}\right)=\int_{T_{\mathrm{ads}}}^{T_{\mathrm{s}}} \Delta H d\left(\frac{1}{H}\right) .
$$

Supposant $L$ et $\Delta H$ constants (ce qui est une bonne approximation puisque l'on est à $\theta$ constant), on obtient :

$$
L\left(\frac{1}{T_{\mathrm{ev}}}-\frac{1}{T_{\text {cond }}}\right)=\Delta H\left(\frac{1}{T_{\mathrm{ads}}}-\frac{1}{T_{\mathrm{S}}}\right) .
$$

TEMPÉRATURES COUPLÉES. - Le raisonnement effectué sur la partie AB du cycle vaut également pour la partie $C D$ du cycle (Fig. 5) puisqu'à nouveau nous serons à $\theta$ constant et que $\Delta H$ sera constant. On obtient donc pour $T_{\mathrm{s}^{\prime}}$, la température couplée à la température de régénération :

$$
L\left(\frac{1}{T_{\mathrm{ev}}}-\frac{1}{T_{\text {cond }}}\right)=\Delta H\left(\frac{1}{T_{\mathrm{S}^{\prime}}}-\frac{1}{T_{\mathrm{reg}}}\right) .
$$

COEFFICIENT DE PERFORMANCE. - Nous avons introduit le coefficient de performance thermodynamique $C_{0}=L / \Delta H$, d'après l'équation (a.17) nous voyons que :

$$
C_{0}=\frac{L}{\Delta H}=\frac{\frac{1}{T_{\mathrm{S}^{\prime}}}-\frac{1}{T_{\mathrm{reg}}}}{\frac{1}{T_{\mathrm{ev}}}-\frac{1}{T_{\text {cond }}}}
$$

$C_{0}$ n'est autre que le coefficient de Carnot calculé avec les quatre températures de fonctionnement $T_{\text {reg }}, T_{\mathrm{S}^{\prime}}, T_{\text {cond }}, T_{\text {ev }}$ alors qu'une machine tritherme donne le coefficient de Carnot avec les quatre températures $T_{\text {reg }}, T_{0}, T_{0}, T_{\text {ev }}$. Par ailleurs d'après (a.7) $C_{0}$ s'écrit également :

$$
C_{0}=\frac{T_{\text {cond }}}{T_{\text {reg }}} \frac{1}{1+\frac{T \Delta S_{\mathrm{e}}}{L}} .
$$

Le terme $T \Delta S_{\mathrm{e}}$ étant très petit on a donc :

$$
C_{0} \sim \frac{T_{\text {cond }}}{T_{\text {reg }}} .
$$

La variation de $C_{0}$ en fonction de $T_{\text {reg }}$ est représentée sur la figure 3 .

\section{Bibliographie}

[1] Meunier, F., Cahier AFEdES no 5 (1978).

[2] Meunier, F., MisChler, B., ISES Conference Atlanta, 28 mai1 er juin 1979.

[3] Simonot Grange, M. H. et Guilleminot, J. J., C. R. Hebd. Séan. Acad. Sci. 287C (1978) 305.

Guilleminot, J. J., Thèse Dijon.

[4] Meunier, F., Mischler, B., Guilleminot, J. J., Simonot Grange, M. H., ISES Conference Atlanta, 28 mai1er juin 1979.

[5] Physical Adsorption of Gases, Young and Crowell (Butterworths) 1962.
[6] Barrer, R. M. et Reucroft, P. J., Proc. R. Soc. A 258 (1960) 431 (Part I) et références jointes.

[7] Bering, B. P., Dubinin, M. M., Serpinskir, V. V., J. Colloid. Interface Sci. 21 (1966) 378.

[8] AshraE, Handbook of Fundamentals (1977).

[9] Rhône-Poulenc, Communication privée.

[10] Cointot, A., Cruchaudet, J. et Simonot-Lagrange, M. H., Bull. Soc. Chim. Fr. 2497 (1970).

[11] Simonot-Grange, M. H., Guilleminot, J. J., Setier, J. C. et Meunier, F., Preprint. 